\title{
Área de Humanidades y Ciencias Sociales: una visión en el tiempo
}

\author{
$M^{a}$ Asunción Martín Lou \\ Con la colaboración de María Isabel Bodega, Ascensión \\ Calatrava, Margarita Delgado, $M^{a}$ Angeles Durán, \\ Valentina Fernández Vargas, Elvira Gangutia, \\ Concepción Roldán, Rosario Sevilla
}

Arbor CLXXII, 679-680 (Julio-Agosto 2002), 537-548 pp.

La intención de mi colaboración en este número de la Revista Arbor dedicado a "Ciencia y Tecnología en el CSIC: una visión de género», no es hacer un análisis exhaustivo del área y de su problemática de género. Más bien voy a dedicar estas líneas a realizar un breve relato histórico y un análisis puntual, a modo de foto fija, del momento actual, en base a la información recogida en diferentes documentos del CSIC para los datos cuantitativos y, lo que es más importante, en base a las experiencias personales de científicas (entre las que incluyo la propia después de treinta y seis años en el organismo), con diferentes orígenes académicos, que se dedican también a distintas líneas de investigación en los Institutos que componen el área. A todas ellas les doy las gracias y citaré sus valiosas y acertadas opiniones.

Un estudio de género que permita ver el cómo y el cuándo se producen los principales acontecimientos en la vida profesional de las mujeres que se incorporan a la vida laboral de la investigación, requiere un análisis largo en el tiempo y profundo en cuanto a los múltiples indica- 
dores que forman parte de esa trayectoria vital. Pero no es el momento de hacerlo sino el de, como dice el título, dar una visión de género.

Sin embargo las especialistas en estos temas de género, están en la idea de esta profundización y no dudo de que llegarán al final.

El Área de Humanidades y Ciencias Sociales (HH. y CC. SS.) del Consejo Superior de Investigaciones Científicas está integrada por diecinueve institutos, de los cuales trece tienen una estructura propia, es decir, dependen exclusivamente del CSIC y el resto seis, tienen una estructura compartida con otros organismos o administraciones. El origen de algunos de estos institutos es anterior al nacimiento del CSIC el 24 de noviembre de 1939 .

Localización de los centros CSIC en HH.CC.SS.

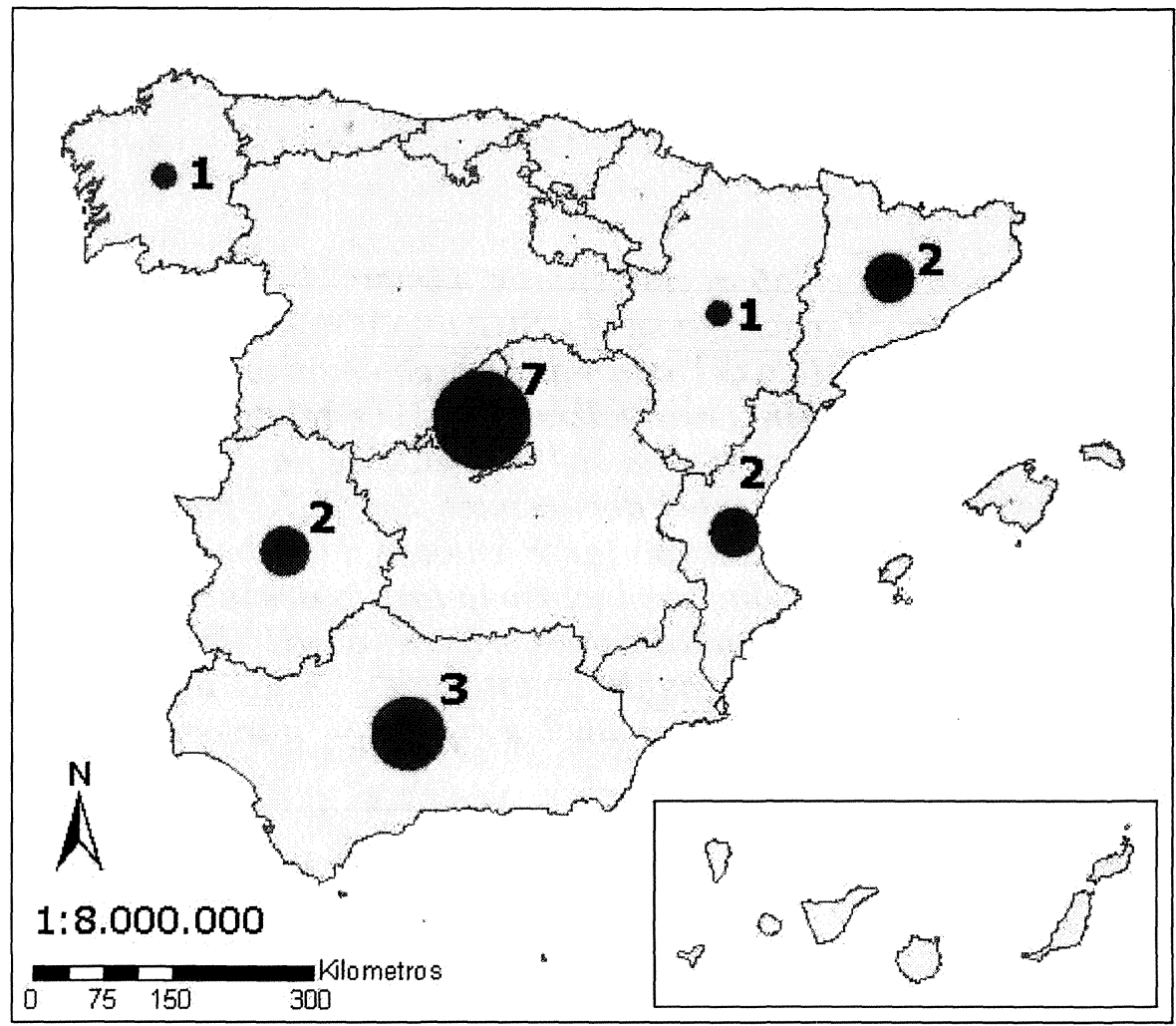

En efecto, la Junta de Ampliación de Estudios, por lo que se refiere al Área científica que nos ocupa, aglutinaba a investigadores en: filología, 


\section{Área de Humanidades y Ciencias Sociales}

\begin{tabular}{|c|c|}
\hline CENTROS & SIGLAS \\
\hline C.A. DE ARAGÓN & \\
\hline Instituto de Estudios Islámicos y del Próximo Oriente & IEOP \\
\hline C.A. ANDALUZA & \\
\hline Escuela de Estudios Arabes & EEA \\
\hline Escuela de Estudios Hispánicos & EEHA \\
\hline $\begin{array}{l}\text { Instituto de Estudios Sociales Avanzados de Andalucía } \\
\text { C.A. CATALANA }\end{array}$ & IESAA \\
\hline Institución Milá y Fontanals & IMF \\
\hline Instituto de Análisis Económico & IAE \\
\hline C.A. GALLEGA & \\
\hline $\begin{array}{l}\text { Instituto de Estudios Gallegos Padre Sarmiento } \\
\text { C.A. DE EXTREMADURA }\end{array}$ & IEHPS \\
\hline Instituto de Arqueología & \\
\hline Instituto Histórico Hoffmeyer & $\mathrm{IHH}$ \\
\hline C.A. DE MADRID & \\
\hline Centro de Información y Documentación Científica & CINDOC \\
\hline Instituto de Lengua Española & ILE \\
\hline Instituto de Economía y Geografía & IEG \\
\hline Instituto de Filosofía & IFS \\
\hline Instituto de Historia & $\mathrm{IHH}$ \\
\hline Unidad de Políticas Comparadas & UPC \\
\hline Instituto de Filología & IFL \\
\hline C.A. VALENCIANA & \\
\hline Instituto de Gestión de la Innovación y del Conocimiento & INGENIO \\
\hline Instituto de Historia de la Ciencia y Documentación López Piñero & $\mathrm{IHCD}$ \\
\hline ROMA (ITALIA) & \\
\hline Escuela Española de Historia y Arqueología & \\
\hline
\end{tabular}

historia, arqueología y arte, en el Centro de Estudios Históricos de la calle de Medinaceli y, más allá de nuestras fronteras, en Roma, la Escuela Española de Historia y Arqueología (Gutiérrez Ríos, 1970).

Esta labor de fomento de la investigación y de promoción de la ciencia y la cultura en general que llevó a cabo la Junta de Ampliación de Estudios, podemos decir que fue el origen del CSIC no solo en el área científica que nos ocupa, sino de la mayoría de las que se organizaron para dar estructura al naciente organismo público de investigación. Así pues, este 
nuevo organismo científico no partía de cero, y aunque estos primigenios núcleos de investigación se ubicaban mayoritariamente en Madrid, desde el inicio «el CSIC no es un punto sino un mapa», y la difusión geográfica de sus centros fue la primera tarea a la que se dedicó el Secretario General, J.M. Alvareda, a fin de buscar y aglutinar por toda la geografía española personas o instituciones con posibilidades para la investigación. (Gutiérrez Ríos, 1970).

No todos los institutos han tenido en el tiempo y en el espacio el mismo origen. Algunos de los actuales institutos surgen con la especialización de determinadas disciplinas, o con la necesidad de profundizar en aspectos de nuestra sociedad y de nuestra historia.

Los diecinueve Institutos del área están repartidos irregularmente por la geografía española, la mayoría de ellos en la Comunidad de Madrid, pero también localizados en otras Comunidades Autónomas como en Andalucía, Aragón, Cataluña, Extremadura, Galicia y Valencia y, como decía, uno de ellos, en Roma.

\section{Difícil renovación generacional}

En relación al número de institutos que componen el Área de conocimiento que nos ocupa, cabría esperar, que también los investigadores fueran numerosos. Sin embargo del conjunto de áreas de investigación en el que está distribuido el personal del CSIC, solamente 215 científicos integran el Área de HH. y CC. SS., de ellos el 34,9\% son mujeres. Por el contrario, en relación al género, junto con el Área de Ciencias Agrarias $(37,7 \%)$ y Ciencia y Tecnología de Alimentos $(39,8 \%)$ tiene los mayores porcentajes de investigadoras.

Entre 26 y 65 años, se sitúa el total del colectivo que estamos analizando, con un predominio de investigadoras en la etapa, de vida profesional, comprendida entre los 46 y los 65 años, edad, esta última, de jubilación voluntaria. Solamente 18 investigadoras tienen menos de 45 años, lo que nos ofrece una perspectiva de madurez peligrosamente alta, si pensamos en la necesaria renovación generacional. Este grupo de edad representa el $70 \%$ sobre el total del área, lo que se aleja mucho del $49 \%$ de este mismo tramo de edad, en el total del CSIC.

Este dato cuantitativo que pone en peligro, como decía, la renovación generacional del colectivo científico del Área de HH. y CC. SS., tiene mucho que ver, sin duda, con la Oferta de Empleo Público que, aunque con un constatable aumento en las convocatorias de 1999 y 2000 en relación a años anteriores, ha supuesto la incorporación de solamente 38 científi- 
cas a la plantilla del CSIC en este último año, 2000, de las que tan solo dos lo han hecho al área de HH. y CC. SS. Este último dato puede sumarse al ya citado en relación a la mayoría de investigadoras en el tramo de edad 45-65 años, con lo que vemos reforzada esa idea de la dificultad de renovación de la plantilla de científicas en nuestra área.

Las setenta y cinco investigadoras están irregularmente repartidas en las tres escalas de la estructura científica. Solamente seis son profesoras de investigación, diecinueve son investigadoras científicas y cincuenta son científicas titulares. Estas cifras absolutas se corresponden con unos porcentajes del $20 \%$, $37 \%$ y $38 \%$ respectivamente que, como vemos en el cuadro I representan unos porcentajes superiores a los que presentan estas mismas cifras para el total del CSIC en el caso de los dos primeros escalones y un punto de diferencia respecto al primer tramo de la carrera profesional, es decir el de científicos titulares.

\section{CUADRo I}

\begin{tabular}{|l|c|c|c|c|}
\hline Escala & $\begin{array}{r}\text { Total Área } \\
\text { HH. y CC.SS. }\end{array}$ & $\begin{array}{c}\% \text { de mujeres } \\
\text { sobre total } \\
\text { Área }\end{array}$ & $\begin{array}{c}\% \text { de mujeres } \\
\text { sobre total } \\
\text { CSIC }\end{array}$ & Total CSIC \\
\hline Prof. de investigación & 30 & 20 & 13 & 332 \\
Investigador Científico & 51 & 37 & 26 & 520 \\
Científico titular & 132 & 38 & 37 & 1.267 \\
TOTAL & 215 & 34 & 30 & 2.119 \\
\hline
\end{tabular}

Hasta aquí la presentación cuantitativa de algunos de los indicadores que reflejan la estructura del colectivo de científicas en el área de HH. y CC. SS.: algo más de un tercio del personal científico del área, con un predominio de edades cercanas al final de la vida laboral, pero con una distribución por categoría profesional que, en absoluto se puede menospreciar si la comparamos con la que ofrece la del total del CSIC.

\section{La visión de género de HH. y CC. SS. en la historia del CSIC}

«... La ciencia se ha construido socialmente y sus contenidos son, consecuentemente, parciales. La experiencia histórica de hombres y mujeres ha sido distinta y la ausencia de las mujeres en los procesos de creación y 
codificación del conocimiento ha dejado su huella en todos los ámbitos del saber» ( $\mathrm{M}^{\mathrm{a}} \mathrm{A}$. Durán).

Atendiendo a la categoría de "género» lo haremos teniendo en cuenta, como cita Inés Alberdi en palabras de Giddes, «... tenemos que hacer una distinción fundamental entre género y sexo. Mientras que el sexo se refiere a las diferencias fisicas del cuerpo, el género alude a las diferencias psicológicas, sociales, culturales entre hombres y mujeres» (Alberdi, 1999). Es, en estas circunstancias, a las que añadiría las de política científica, en donde vamos a encontrar las diferencias en las trayectorias profesionales de hombres y mujeres de quienes integran el área y también las diferencias que puedan existir con otras áreas científicas del CSIC, que se analizan en otros trabajos de este número de Arbor.

¿Cuál ha sido la trayectoria personal-profesional de las científicas pertenecientes al área de HH. y CC. SS.? ¿Existe diferenciación en esta trayectoria profesional en razón de género?

Después de escuchar y leer las aportaciones a que antes aludía, sumadas a mi propia experiencia, no se percibe claramente un sesgo por razón de género, aunque se plantean muchos interrogantes en cuyas respuestas existen múltiples circunstancias. Estas circunstancias no sólo responden a la historia de los propios institutos sino también a los diferentes puntos de partida de cada una de las científicas y que son a su vez consecuencia de sus propias singularidades familiares, económicas, etc.

En la primera etapa de formación del CSIC, se integraban en los institutos que se iban configurando una serie de catedráticos universitarios, grandes maestros que compaginando su labor docente universitaria con la vocación investigadora, tuvieron la posibilidad de formar y contar con personas jóvenes dotadas con becas que, aunque ahora parecerían míseras, posibilitaron el acceso a una infraestructura de investigación que permitió emerger del aislamiento científico y conectar a estos incipientes institutos con el resto del mundo (E. Gangutia). Nombres ilustres en la ciencia española ocuparon los cargos directivos de los institutos en este inicio del CSIC: Menéndez Pidal, Asin, Ballesterọs, Entrambasaguas, Viñas y un largo etc.

Esta primera etapa de nuestra Área, está ligada estrechamente a las universidades, de forma que los universitarios que iniciaban su vida profesional en el seno del CSIC muy pronto pasaban a ocupar puestos de plantilla en departamentos universitarios, mientras que las mujeres, sus compañeras, permanecían en el CSIC. Esta es una experiencia vivida por mí y que, supongo, comparto con otras investigadoras lectoras de esta líneas que reconocerán, en algunos catedráticos universitarios, compañeros del CSIC con los que se iniciaron en la carrera profesional. También 


\section{Área de Humanidades y Ciencias Sociales}

existen las excepciones como la de $\mathrm{M}^{\mathrm{a}}$ Angeles Galino, primera catedrática de España, que se formó en el Instituto Suárez. En los años sesenta, cuando ya se percibían otras formas, $\mathrm{M}^{\mathrm{a}}$ Eugenia Aubet, pasó de investigadora científica a catedrática, y aunque desde el principio se han producido pasos en esa dirección, creo que la migración femenina hacia la universidad ha sido escasa.

Siguiendo con este breve repaso histórico de los institutos del Área de $\mathrm{HH}$. y CC. SS. focalizado espacialmente en la C/ Medinaceli (Madrid), en donde estuvieron y están los principales institutos de Humanidades, sin menospreciar los que se localizan en otras Autonomías pero que son posteriores, hay que destacar que también allí surgieron los primeros núcleos de investigación en ciencias sociales, que aunque carecieron, como tantos otros, de personal científico de plantilla hasta 1971, son los artífices del progreso de esta área de investigación en una sociedad en la que se abrían paso no sin dificultades.

La historia del Instituto Balmes y de sus publicaciones (1944 aparece ya la Revista Internacional de Sociología editada por el Instituto) son el fiel reflejo de esta situación. Es también el Instituto Balmes el que por primera vez cuenta con una directora, Valentina Fernández Vargas, que dirigió igualmente la RIS y otras publicaciones, que recogían las investigaciones más importantes de esta área de conocimiento. En el Instituto se hacia especial énfasis en el estudio y difusión del análisis demográfico, razón por la que Corrado Gini fue codirector con Severino Aznar, uno de los máximos representantes de la sociología católica.

Con la desaparición de los Patronatos y las Divisiones, estructura mantenida durante muchos años en el CSIC, en la que todas las nuevas áreas temáticas que aparecían tenían cabida, desaparecieron también institutos emblemáticos que en el mejor de los casos dieron lugar a Departamentos dentro de los actuales Institutos. Es el caso del Balmes, repartidas sus líneas de investigación en el momento actual en el Instituto de Economía y Geografía, Unidad de Políticas Comparadas e Instituto de Estudios Sociales Avanzados de Andalucía.

Otras áreas temáticas incluidas dentro del Área han tenido procesos y génesis diferentes. Así el Instituto de Estudios Jurídicos que desapareció de la estructura del CSIC en la década de los setenta, o el Instituto de Economía «Sancho de Moncada» que dio origen al Instituto de Economía Aplicada, ubicado en la UAM. También Geografía, ciencia a caballo entre las humanidades y las ciencias sociales, entonces y aún ahora, tuvo, desde la creación del CSIC, un lento pero continuo crecimiento en dos orientaciones que, aunque no antagónicas, re- 
presentaban dos concepciones de la disciplina: una más histórica y humanista encabezada por el Prof. Manuel de Terán y otra más aplicada a las necesidades de la sociedad y del análisis territorial que desarrolló la escuela del Prof. José M. Casas Torres. Y para no ser distintos, ambos eran catedráticos universitarios y nunca pertenecieron a la plantilla del CSIC. Otros institutos fueron ampliando el número de los que componen el área, dedicándose a líneas de investigación no desarrolladas hasta hace unos cuantos años en el CSIC. Estos movimientos, no han dejado de producirse. Así, por ejemplo, el Instituto de Demografía que nació en 1988 con una gran proyección social, fue convertido en Departamento del mismo nombre en 1996 anexionado al Instituto de Economía y Geografía. El Centro de Estudios Históricos acaba de sufrir una última reestructuración, agrupándose en una única estructura administrativa y en tres Institutos (Lengua, Historia y Filología) las múltiples líneas de investigación que desde la época de la Junta de Ampliación de Estudios se desarrollan en Medinaceli. Y a la vez que se funden líneas de investigación en un único instituto, otros nuevos núcleos científicos aparecen en nuestra estructura, tal es el caso del Instituto Histórico Hoffmeyer o el de Estudios Islámicos y del Próximo Oriente.

Puede decirse que en el momento actual, a excepción hecha ya del área del Derecho, todas las áreas temáticas de las humanidades y ciencias sociales tienen su lugar en el CSIC: Historia, Arte, Filosofía, Arqueología, Economía, Geografía, Sociología, Demografía, Musicología, Historia de la Ciencia y del Pensamiento, Estudios Locales, ... etc., aunque con diferente plasmación en la estructura del organismo.

Merece destacarse que en esta primera fase de desarrollo del CSIC en la que los institutos estuvieron estrechamente ligados a la universidad, las mujeres que en ellos trabajaron, y no solo las científicas, contribuyeron de manera decisiva al crecimiento del área. No solo por los trabajos de investigación, en muchas ocasiones tesis doctorales, sino también porque la dedicación a sus institutos permitió la formación y organización de las bibliotecas, la gestión y edición de las revistas científicas del área, entre las que se encuentran las de mayor prestigio de España y que, a través de sus intercambios, constituyen ahora un patrimonio fundamental para nuestra área.

Es de justicia destacar en este punto, la labor que han desempeñado y desempeñan las numerosas y valiosas mujeres que se implicaron en estas tareas auxiliares imprescindibles para la investigación. Todo el personal científico del Área estamos obligados a reconocer esta labor, aunque no sea este espacio el destinado para analizarla. 
Pasada esta primera etapa, a partir de los años sesenta, se inicia un proceso en el que la investigación se financia a través de proyectos. Se termina el paternalismo que caracterizó la etapa anterior y la investigación en equipo será desde entonces, salvo excepciones, la forma habitual de investigar. Ello llevó, inicialmente en nuestro ámbito, a una integración y después a un despegue de la investigación de las mujeres, por la posibilidad naciente de tener financiación independiente, de desarrollar proyectos propios, con un protagonismo similar al de los investigadores varones y en un momento en el que el CSIC se equiparaba, en prestigio, a la Universidad (E. Gangutia).

$\mathrm{El}$ proceso histórico que se ha planteado en las líneas precedentes, ha sido la reciente historia de los Institutos de nuestra Área. Claro está, que aquellos que tienen un origen más reciente, se han incorporado ya a la etapa en la que la independencia investigadora preside, como norma general nuestro trabajo. Pero teniendo en cuenta, como hemos visto, la edad de las científicas del área, la mayoría de nosotras hemos pasado por esta etapa inicial "paternalista» y la posterior, de independencia investigadora, ya en plena madurez científica.

Al final de la década de los sesenta y primeros de los setenta, cuando la formación universitaria para las mujeres deja de ser un indicador de excepcionalidad y muchas, de las hoy investigadoras de nuestra área, comienzan su vida laboral, el formar una familia propia constituía también una forma de independencia de la familia tradicional. Conseguir esta libertad, solo aparente, y conciliar el trabajo de investigación con la formación de la familia sería, en la mayoría de los casos, un obstáculo para tener un proceso profesional con los mismos tiempos y resultados que alcanzaban los investigadores varones. ( $\mathrm{M}^{\mathrm{a}} \mathrm{I}$. Bodega).

Aunque no fue lo más habitual, en algunos casos, esta dedicación a la familia o la participación en trabajos de los institutos, no estrictamente de investigación, retrasaron la ejecución de la tesis a más de una de las actuales colegas investigadoras. Puede decirse que estas circunstancias fueron obstáculos en razón de género (encubierto) debido a las necesidades personales de dedicación a la familia o, la necesidad económica de mantener un puesto de trabajo. «... no tengo claro todavía si los problemas que fueron surgiendo a lo largo de mi carrera profesional y que pudieron solventarse, ocurrieron por mi condición de mujer o fueron independientes de este hecho específico» (A. Calatrava).

«... recuerdo los años duros de inserción en el mercado de trabajo, la difícil compatibilización con la vida familiar. Durante muchísimos años no he tenido vacaciones. Los libros, la memoria de cátedra y los principales informes los he escrito de madrugada, en el silencio acogedor de mi 
mesa de trabajo. Fuera de la familia y el estudio o la investigación quedaba muy poco sitio para otras cosas, para mi misma ...» ( $\mathrm{M}^{\mathrm{a}} \mathrm{A}$. Durán).

\section{El poder lo ostentan los hombres}

Aunque en el momento actual seis de los institutos del área están dirigidos por mujeres (CINDOC, EEHA, IEG, IFL, IH, IHH), son los hombres los que dominan las cifras de nuestro entorno científico.

Es curioso observar como esta discriminación numérica se arrastra en la configuración de los Tribunales, tanto en los de oferta de empleo público como en los de promoción interna. Solamente en los formados para juzgar la promoción de Titulados Superiores Especializados encontramos un $60 \%$ de mujeres en la composición de los mismos. Por el contrario, cuanto más elevado es el nivel a juzgar, menos es el porcentaje femenino de los componentes de los tribunales: Profesores de Investigación $13 \%$, Investigadores Científicos $16 \%$ y Científicos Titulares $24 \%$.

Lo habitual es que sean hombres los directores de tesis, los integrantes de tribunales, tanto de tesis como de oposiciones, los directores de equipos de investigación, y aunque según la percepción de la mayoría de las investigadoras consultadas no está claro que estos hechos sean debidos a una discriminación en razón de género, las cifras están ahí, y las diferencias podemos decir que son más bien consecuencia de cuestiones relacionadas con la política ... científica.

«... en mi trabajo científico en el CSIC no me siento en absoluto discriminada, aunque considero que mi trayectoria profesional se encuentra un tanto frenada, pero no estoy segura (aunque alguna vez lo he pensado) de que ello se corresponda con el hecho de ser mujer, sino más bien a cuestiones más generales de política científica»(M. Delgado).

De este olvido hacia las mujeres científicas está llena la etapa paternalista a la que antes aludíamos. Solamente circunstancias personales, casi siempre ligadas a la familia, han logrado vencer esta circunstancia.

Entre las experiencias personales aportadas por investigadoras del área reproduzco ahora la de Concha Roldán, una reciente (1997) investigadora del área perteneciente al Instituto de Filosofía. No es vanal incluirla aquí. Considero que reflejan con firmeza el estado de la cuestión en nuestra área y, más aún, confirma lo que muchas hemos experimentado desde hace más d€ treinta años.

«... Mi trayectoria profesional en el CSIC tampoco consigue zafarse de la jerarquización androcéntrica mencionada en esta institución pública de investigación, en la que desde 1997 soy Científica Titular, después de 


\section{Área de Humanidades y Ciencias Sociales}

una década de vinculación intermitente con la institución como becaria y contratada. Para hacer honor a la verdad he de decir, además, que soy la única mujer investigadora en la plantilla en la joven historia de este pequeño instituto que en la actualidad cuenta ya con catorce investigadores, entre los que desde el principio fue considerada «como uno más», si es que puede emplearse esta expresión sin que suene discriminadora. Indaguemos la causalidad de este hecho: no hay que buscarla precisamente en la escasez de mujeres con un currículum valioso en el campo de la investigación filosófica, algunas de las cuales han sido becarios en nuestro instituto. ¿Se debe entonces a la casualidad este hecho que me hace enfrentarme cada día con una sensación de excepcionalidad?

¿Qué fue, por ejemplo, de mis compañeras de promoción o de promociones anteriores y posteriores a la mía?. Nos licenciamos en una promoción superior al $60 \%$ de los varones, con notas bastantes mejores que ellos en la mayoría de las asignaturas, lo que a muchas nos permitió acceder a distintas becas de investigación. Sin embargo, apenas nos contamos con los dedos de una mano las que conseguimos horadar un hueco en el resistente muro de la Academia, mientras que a muchas sí se les abrió el campo de la Enseñanza secundaria, o incluso primaria, tanto en centros estatales como privados. Algunos "científicos» afirman que la mayoría de las mujeres carecen de la testosterona suficiente para alimentar su ambición profesional y que por eso «se quedan» en puestos subordinados con respecto a los varones «más agresivos». Así de sencillo, como recuerdan las clasificaciones que van desde Aristóteles hasta Linneo: nosotras mismas nos vamos autoexcluyendo por el camino debido a una sencilla «cuestiona hormonal». Parecería obvio que la filosofía, con su consideración como disciplina crítica por excelencia y, sobre todo, con su pasado ilustrado de lucha contra los prejuicios, hubiera debido enfrentarse al problema con otro talante, pero son pocos los filósofos que se han acercado al debate sobre el género de una manera que no sea meramente oportunista o anecdótica. Salvando esas honrosas excepciones, una pequeña parte de nuestros colegas varones están asentados en su cota de poder y no quieren arriesgarse a perderla concediendo de facto el estatus de igualdad a sus colegas mujeres; la mayoría, sencillamente «no nos ve».

\section{Conclusión y perspectivas de futuro}

En las líneas que anteceden he expuesto unas consideraciones, muy generales, con respecto al proceso histórico y a los datos actuales del área de HH. y CC. SS. y de las científicas que la integran. 
Con respecto a la historia, la mayoría de los institutos tienen sus orígenes en los del CSIC y aún lo anteceden como hemos visto en el caso del Centro de Estudios Históricos de Medinaceli.

En cuanto al proceso científico, sigue en paralelo al desarrollo social de nuestro país. También la investigación realizada por mujeres ha logrado una independencia que se manifiesta en una participación equiparable a la de los hombres en cuanto a resultados de la investigación, publicaciones, participación en proyectos, conferencias, seminarios, etc.

No podemos decir lo mismo si comparamos la participación de las científicas en puestos directivos, tribunales ..., en fin, en órganos de decisión a pesar de que, como hemos visto, el porcentaje de mujeres es suficiente para tener una mayor presencia.

¿Es a causa del género el retraso en el tiempo que en muchos casos experimentan las científicas en su trayectoria profesional?

Parece ser, según hemos constatado en las conversaciones mantenidas que no hay conciencia clara de ello. Pero ¿acaso la dedicación a la familia, a los hijos (colegio, médico, etc.), a los padres en la edad actual de la mayoría de nosotras, no es un indicador de género?

¿Y el futuro?. El futuro no podemos tampoco desligarlo de las tendencias generales de nuestra sociedad actual, en donde las HH. y CC. SS. tienen un escaso reconocimiento de su valor y trascendencia. No deben olvidar quienes hacen política científica que nuestras disciplinas recogen el patrimonio histórico y cultural y que, sólo el conocimiento de nuestra sociedad en todas sus manifestaciones permitirá seguir teniendo un lugar importante en el entorno científico que hemos heredado y nos corresponde.

\section{Bibliografía}

Alberdi, I. (1999): «El significado del género en las Ciencias Sociales». Rev. Política y Sociedad, 32 , Madrid (pp. 9-21),

Durán Heras, Ma A. (1991): "Las contradicciones de la sociedad igualitaria». Rev. Investigaciones Psicológicas, 9, Universidad Complutense, Madrid, pp. 167-184.

FERNÁNDEZ VARGAS, V. (1999): “Españolas en la transición». Eds.: Asociación MUJERES EN LA TAANSICIÓN DEMOCRÁTICA. Ed. Biblioteca Nueva, pp. 427-450.

GutiérRez Ríos, E. (1970): José $M^{a}$ Albareda. Una época de la cultura española. Madrid, CSIC, 342 p. 